\title{
ESTUDO EXPERIMENTAL DE ESTABILIDADE DE CHAMAS DIFUSIVAS DE GÁS NATURAL DILUÍDAS COM DIÓXIDO DE CARBONO SUJEITAS A ESCOAMENTO CRUZADO
}

\author{
Manuel A. K. Nzinga ${ }^{(1)}$ (kionynzinga1958@gmail.com), Felipe Roman Centeno ${ }^{(2)}$ (frcenteno@ufrgs.br), \\ Fernando Marcelo Pereira ${ }^{(3)}$ (fernando@mecanica.ufrgs.br) \\ (1) Universidade Federal do Rio Grande do Sul (UFRGS); Departamento de Engenharia Mecânica
}

RESUMO: Esta pesquisa descreve o estudo experimental de chamas turbulentas difusivas de gás natural veicular diluídas com dióxido de carbono, sujeitas a vento cruzado. Para estudar a estabilidade (geometria) foi montado um aparato experimental a fim de realizar os experimentos. Os resultados experimentais obtidos sobre a geometria da chama dizem respeito ao comprimento de chama e o ângulo de inclinação da chama. $A$ adição de dióxido de carbono na mistura com o combustível e do vento cruzado ocasionou a redução do comprimento de chama, enquanto que, para o ângulo de inclinação da chama, a velocidade do vento cruzado teve maior influência quando comparado com o dióxido de carbono.

$\prod$

PALAVRAS-CHAVE: chamas turbulentas difusivas, vento cruzado, efeito de dióxido de carbono.

\section{EXPERIMENTAL STUDY OF STABILITY OF DIFFUSIVE FLAMES OF NATURAL GAS DILUTED WITH CARBON DIOXIDE SUBJECTED TO CROSSED FLOW}

ABSTRACT: This work describes the experimental study of turbulent diffusive flames of natural gas diluted with carbon dioxide subjected to crossed wind. To study the stability (geometry) and conduct the experiments an experimental apparatus was set. The experimental results obtained about the geometry of the flame are concerned with the length and the angle of slope of the flame. The addition of carbon dioxide in the mixture with the fuel and of the crossed wind resulted in the reduction of the flame length, while that for the angle of slope of flame the velocity of the crossed wind had more influence when compared to carbon dioxide.

KEYWORDS: turbulent diffusive flames, crossed wind, effect of carbon dioxide.

\footnotetext{
${ }^{1}$ Kionynzinga1958@gmail.com
} 


\section{XIX \\ CENEMI}

\section{INTRODUÇÃO}

Os queimadores a jato são caracterizados pela elevada quantidade de movimento na direção axial e elevada potência, estes queimadores são muito empregados em fornos rotativos, principalmente na indústria de cimento, secagem e da calcinação. Com o desenvolvimento industrial, o uso da queima de combustíveis injetados em um escoamento de ar, ganhou substancial importância no desenvolvimento socioeconômico, influenciando a sociedade e atraindo interesse devido a sua aplicação na secagem de grãos, plataformas de petróleo e extração de petróleo, por exemplo, é de extrema importância, seja em questões ambientais, econômicas ou de segurança.

Johnson e Kostiuk (1999), estudaram os efeitos de escoamento do vento cruzado e de gases diluentes na eficiência do processo de combustão de chamas difusivas. Neste estudo apresentado, eles utilizaram misturas de gás natural diluídas com dióxido de carbono e verificaram uma drástica redução da eficiência de queima com o aumento da velocidade do vento cruzado e com o aumento da fração de gás inerte na mistura. O foco do estudo foram chamas com baixa razão de quantidade de movimento entre os jatos (combustível e vento cruzado).

Kostiuk et al. (2000) propuseram uma escala semi-empírica do comprimento de chamas difusivas em relação à velocidade do vento cruzado, à velocidade do jato (combustível) e ao diâmetro do bocal do queimador. Nos experimentos, utilizaram o gás propano como combustível, mas mantiveram baixa razão de quantidade de movimento entre os jatos. Mais tarde, Majeski et al. (2004) continuaram com o mesmo estudo, assumiram a chama como sendo a superfície de um cilindro inclinado, onde há a mistura estequiométrica com combustível no lado interno e oxidante no lado externo conforme apresentado (Figura 1(b). A fim de melhor estimar o comprimento de chama, foram incluídos parâmetros geométricos e estequiométricos no modelo proposto. Além disso, incluíram um fator representativo de efeitos da diluição de gases inertes na mistura de combustível. Os dados experimentais apresentados foram de acordo com as tendências apresentadas pelo modelo.

Wang et al. $\left(2015^{\mathrm{a}}\right)$ conforme a Figura 1 (b), investigaram a evolução do comprimento de chamas turbulentas difusivas de propano com escoamento de vento cruzado. Nos experimentos, foram variados o diâmetro do queimador, a velocidade do vento cruzado e a pressão atmosférica (64 e $100 \mathrm{kPa}$ ). No entanto, foi observada a redução do comprimento de chama com o incremento tanto 


\section{XIX \\ CENEMI}

da velocidade do vento cruzado quanto da pressão atmosférica. Já com o incremento do diâmetro do queimador, foi verificado o aumento do comprimento de chama.

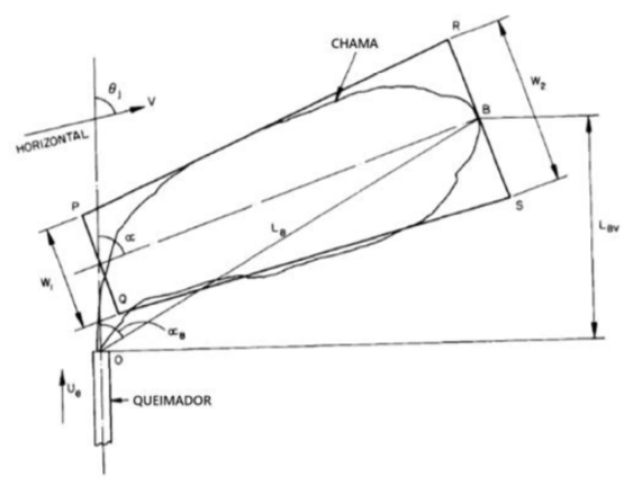

(a)

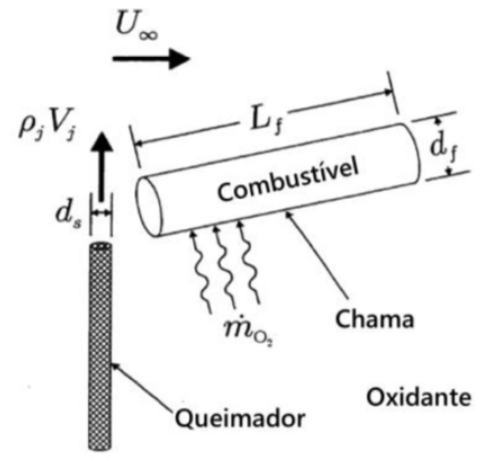

(b)

Figura 1 - a) Modelo de tronco de cone proposto por Kalghatgi, 1983. (b) Modelo proposto por Majeski et al., 2004.

Wang et al. $\left(2015^{b}\right)$ conforme a Figura 1(b) propuseram uma correlação entre o comprimento de chama de acordo os parâmetros físicos e químicos variados nos testes. Na mesma série de experimentos, os autores, analisaram o limite de extinção da chama e observaram que a velocidade do vento cruzado critica a extinção da chama reduz consideravelmente com a diminuição da pressão atmosférica.

A partir das questões mencionadas na literatura, atendendo a contribuição dos autores, este trabalho tem como objetivo estudar a estabilidade bem como as características geométricas de chamas difusivas turbulentas de gás natural veicular, com 7,55 mm de diâmetro interno do bocal do queimador e proporções de diluição de dióxido de carbono com diferentes velocidades de vento cruzado. 


\section{XIX \\ CENEMI}

\section{MATERIAIS E MÉTODOS}

A Figura 2 ilustra a configuração da bancada experimental para o estudo da estabilidade (geometria) da chama.

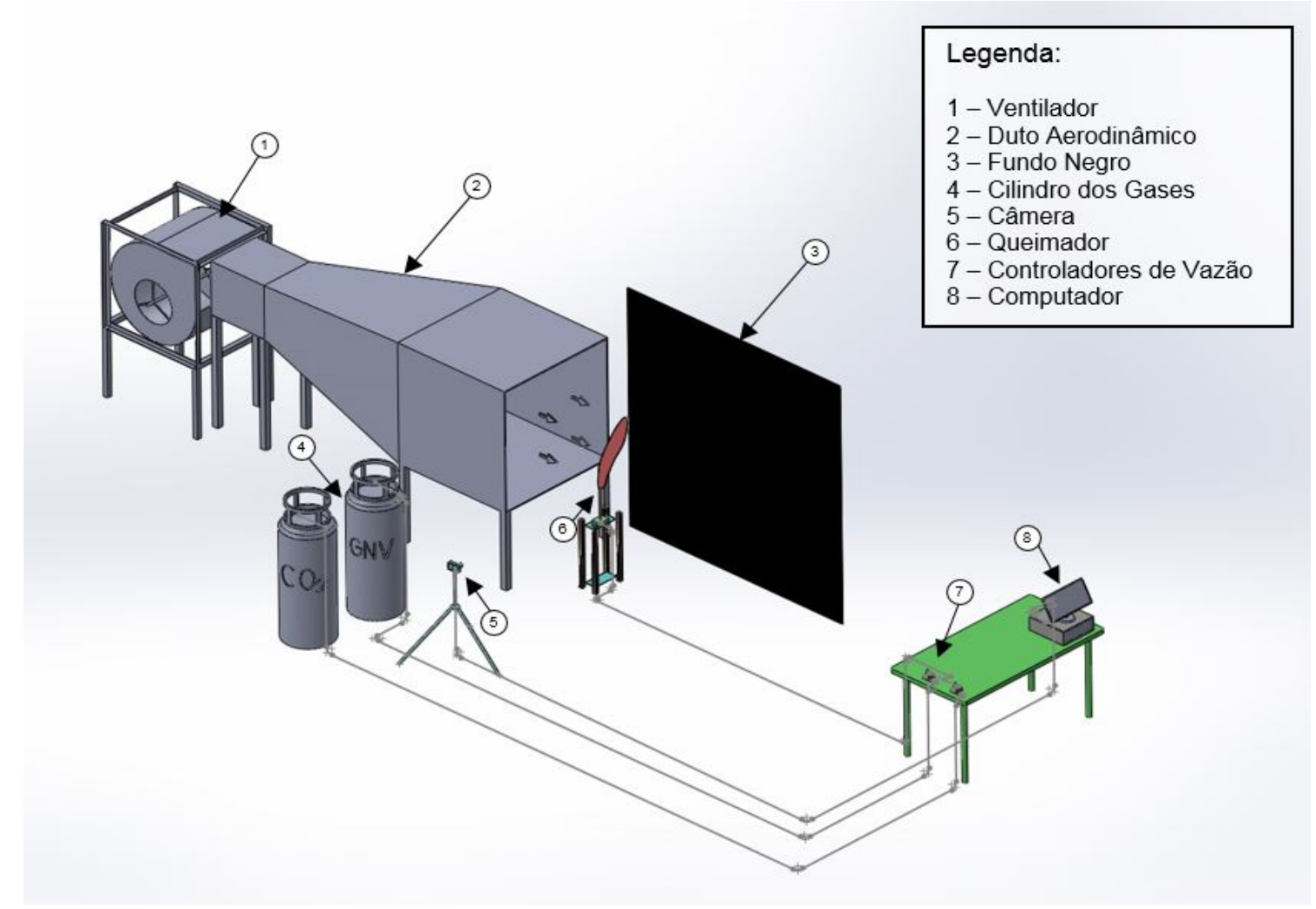

Figura 2 - Esquema ilustrativo da bancada experimental da geometria da chama

Os cilindros de gás natural e de dióxido de carbono em (4) são conectados por tubos a controladores eletrônicos de vazão volumétrica da escala de medição de marca Omêga (FMA-2600A) para o combustível com incerteza de $\pm 0,8 \%$ da leitura e $\pm 0,2 \%$ da escala de medição em (7) e estes, por sua vez, conectados por tubos (PU) que se unem, misturando os gases elevando-os a um queimador de tubo simples com diâmetro interno de 7,55 milímetros com comprimento total do queimador de $30 \mathrm{~d}_{\mathrm{b}}$. Foi utilizado o gás natural veicular comercial GNV como combustível, com composição química aproximada de $90,8 \% \mathrm{CH}_{4}, 6 \% \mathrm{C}_{2} \mathrm{H}_{6}, 1,2 \% \mathrm{C}_{3} \mathrm{H}_{8}, 0,5 \% \mathrm{CO}_{2}$ e 1,55\% $\mathrm{N}_{2}$ [GasNet, 2017]. O dióxido de carbono utilizado possui pureza de 99,2\% e as diluições empregadas foram de 


\section{XIX \\ CIONEMI}

$0 \%, 10 \%, 20 \%$ e $30 \%$ do volume total da mistura, o qual assumiu valores de vazões de mistura (combustível e $\mathrm{CO}_{2}$ ) de 8,63; 13,80; 18,98; 24,15; 29,33; 34,50; 39,68; e 44,86 litros por minuto.

Com o intuito de proporcionar o escoamento de ar uniforme em direção à chama, foi construído um duto aerodinâmico do tipo soprador em (2), composto por três diferentes módulos e acoplado a um ventilador centrifugo em (1). O primeiro módulo possui seção de $0,45 \times 0,45 \mathrm{~m}$ e comprimento de 0,50 m. O segundo módulo possui seção inicial de 0,45x0,45 m, seção final de 1,00×1,00 m e comprimento de 1,50 m. Já o terceiro módulo possui seção de 1,00×1,00 m e comprimento de 1,00 m. Essas dimensões foram projetadas para atender o bocal do ventilador e uma seção de saída capaz de cobrir chamas de até um metro de altura, considerando-se fatores como a perda de carga no túnel, a limitação de espaço no laboratório e o desenvolvimento do escoamento. Além disso, foram instaladas três telas de diferentes tamanhos de malha nas divisas dos módulos, a fim de homogeneizar o escoamento de ar.

O ventilador centrífugo da marca Soler\&Palau foi escolhido em função da perda de carga calculada e da velocidade do vento desejada, e apresenta ponto de operação ótimo em vazão de $10500 \mathrm{~m}^{3} / \mathrm{h}$, pressão de 95,7 mmca e rendimento de 79\%. Um inversor de frequência WEG foi instalado na extremidade do duto aerodinâmico onde está o ventilador, o qual possibilita a variação da frequência de rotação de $0-66 \mathrm{~Hz}$. Para monitorar o cruzamento do fluxo de ar em tempo real, um anemômetro digital foi utilizado, de modelo HOMIS, o qual possui uma faixa de medição de 0,2 a 20 m/s, utilizando como referência conduções atmosféricas, e incerteza de medição de $\pm 5,0 \%$ do valor lido mais $\pm 1,0 \%$ do valor de fundo de escala, sendo instalado verticalmente com intervalos de $10 \mathrm{~cm}$ no portal de saída do túnel. As velocidades do vento cruzado utilizadas no estudo foram de 0,$5 ; 1,0 ; 1,5$ e $2,0 \mathrm{~m} / \mathrm{s}$.

Para realizar as filmagens das chamas, foi utilizada uma câmera fotográfica Canon EOS Rebel T5i em (5). Os parâmetros óticos (abertura do diafragma, tempo de exposição e sensibilidade ISO), com o objetivo de obter imagens bem definidas das chamas. Os parâmetros óticos definidos no estudo são: 4,5; 1/125 s e 1600 a 3200 dependendo da luminosidade da chama. Os experimentos foram realizados com o mínimo de iluminação natural e, adicionalmente, foi instalada uma tela preta opaca em (3) atrás da bancada para proporcionar um contraste adequado nas filmagens. 


\section{XIX \\ CIONEMI}

\section{RESULTADOS E CONCLUSÕES}

\section{Comprimento de chama}

A Figura 4 mostra a evolução do comprimento de chama, $L_{f}$, em relação a vazão de combustível fornecida no jato. Percebe-se o aumento do comprimento de chama com o incremento da vazão volumétrica total de combustível, $\forall$. Com o aumento da diluição de $\mathrm{CO}_{2}$ na mistura e com o aumento da velocidade do vento, $U_{\infty}$, contudo, percebe-se uma diminuição do comprimento de chama, concordando com o que foi reportado por [Wang et al., 2015a]. O incremento da diluição de gás carbônico provoca o aumento da massa específica da mistura e, portanto, o aumento de sua quantidade de movimento, o que poderia resultar no alongamento da chama. Apesar disso, o incremento da diluição representa uma menor quantidade de combustível sendo queimado, resultando no encurtamento da chama.
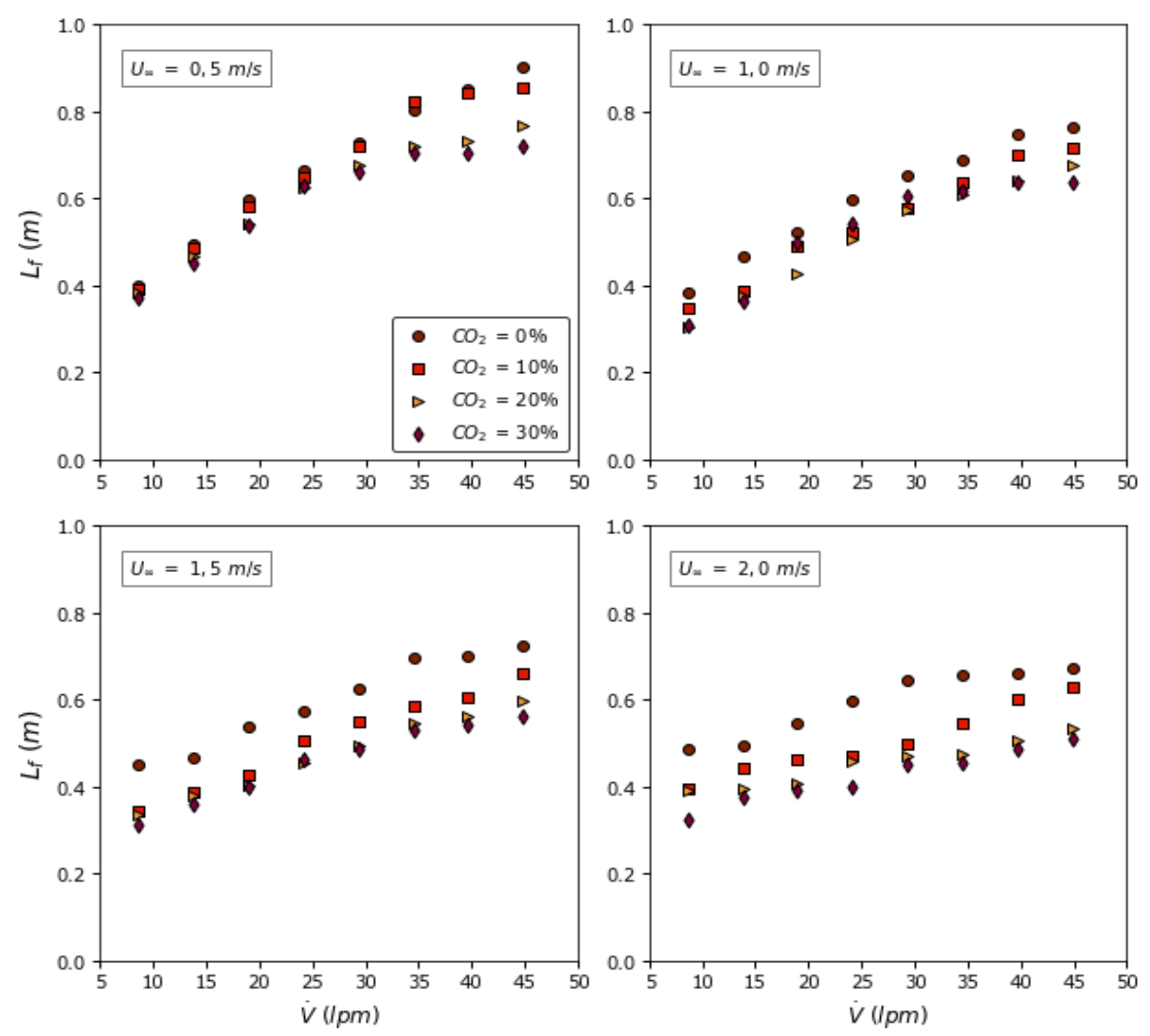

Figura 4 - Variação do comprimento de chama em relação a vazão de combustível no jato.

Como a Engenharia Mecânica e Industrial podem melhorar o Agronegócio do Brasil Anais do XiX CONEMI - Congresso Nacional de Engenharia Mecânica e Industrial | 6 de 9 


\section{XIX \\ CIONEMI}

Ângulo de inclinação da chama

A Figura 5 apresenta a variação do ângulo de inclinação da chama para diferentes valores de vazão de combustível em diferentes diluições de dióxido de carbono (0\% a 30\%) e velocidade de vento cruzado $(0,5 \mathrm{~m} / \mathrm{s}$ a $2,0 \mathrm{~m} / \mathrm{s})$. Observa-se que a inserção de dióxido de carbono na mistura do combustível não gerou alterações significativas nos dois primeiros ventos cruzados $(0,5 \mathrm{~m} / \mathrm{s}$ a 1,0 $\mathrm{m} / \mathrm{s})$, enquanto que para os ventos de $(1,5 \mathrm{~m} / \mathrm{s}$ e $2,0 \mathrm{~m} / \mathrm{s})$, pequenas alterações foram verificadas, próximos testes serão realizados a fim de validar esse efeito. Por outro lado, nota-se que, em cada incremento da velocidade do vento cruzado, o ângulo de inclinação da chama é reduzido nos ventos cruzados considerados, levando a constatação de que a variação da inclinação do ângulo da chama tem maior influência com o vento cruzado do que com a diluição de dióxido de carbono.
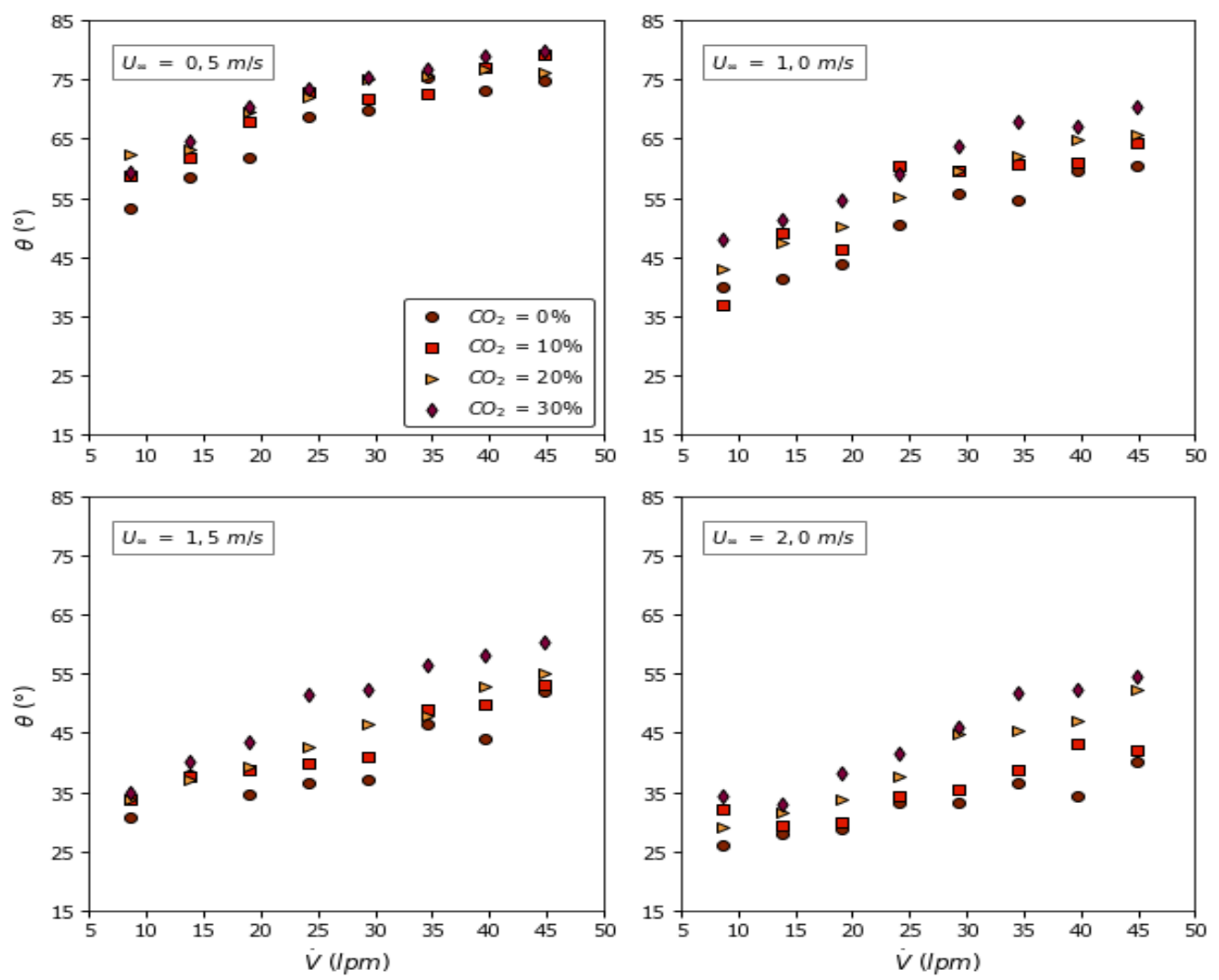

A Figura 5 mostra a variação do ângulo de inclinação da chama, $\alpha$, em relação a vazão de combustível fornecida no jato. 


\section{XIX \\ CONEMI}

A pesquisa se destinou a avaliar quantitativamente o efeito da diluição de dióxido de carbono e da velocidade do vento cruzado em chamas difusivas turbulentas.

Através dos experimentos realizados, foram plotados gráficos com o objetivo de estimar as propriedades geométricas de chamas difusivas de gás natural veicular diluídas com gás carbônico variando a velocidade do vento cruzado. Nos testes, foram estimadas oito potências, de 5 e $26 \mathrm{~kW}$ (incrementadas em três), diluições de $\mathrm{CO}_{2}$ de $0,10,20 \%$ e $30 \%$ e $40 \%$ com velocidades de vento cruzado de 0,$5 ; 1,0 ; 1,5$ e 2,0 m/s. Os resultados experimentais mostraram que o comprimento de chama aumenta com o incremento da vazão de combustível, contudo, ele reduz com a adição da diluição de dióxido de carbono e vento lateral. Da mesma forma, o ângulo de inclinação da chama aumenta com o incremento do combustível e reduz com o incremento da velocidade do vento cruzado. 


\section{XIX \\ CONEMI}

\section{REFERÊNCIAS}

GASNET - O site do gás natural; "Composição do Gás Natural Comercial”, http://www.gasnet.com.br/. Acesso em 20/09/2017.

JOHNSON, M. R.; KOSTIUK, L. W.; "Effects of a Fuel Diluent on the Efficiencies of Jet Diffusion Flames in a Crosswind", Spring Technical Meeting, 1999.

KAlghatgi, G. T.; "The Visible Shape and Size of a Turbulent Hydrocarbon Jet Diffusion Flame in a Cross-wind", Combustion and Flame, vol. 52, pp. 91-106, 1983.

Kalghatgi, G. T.; "Lift-off Heights and Visible Lengths of Vertical Turbulent Jet Diffusion Flames in Still Air", Combustion Science and Technology, vol. 41, pp. 17-29, 1984.

Kostiuk, L. W.; Majeski, A. J.; Poudenx, P.; Johnson, M. R.; Wilson, D. J.; "Scaling of WakeStabilized Jet Diffusion Flames in a Transverse Air Stream", Proceedings of the Combustion Institute, vol. 28, pp. 553-559, 2000.

Majeski, A. J.; Wilson, D. J.; Kostiuk, L. W.; "Predicting the Length of Low-Momentum Jet Diffusion Flames in Crossflow", Combustion Science and Technology, vol. 176, pp. 2001-2025, 2004.

Wang, Q.; Hu, L.; Zhang, X.; Zhang, X.; Lu, S.; Ding, H.; "Turbulent jet diffusion flame length evolution with cross flows in a sub-pressure atmosphere", Energy Conversion and Management, vol. 106, pp. 703-708, 2015a.

Wang, Q.; Hu, L.; Yoon, S. H.; Lu, S.; Delichatsios, M.; Chung, S. H.; "Blow-out limits of nonpremixed turbulent jet flames in a cross flow at atmospheric and sub-atmospheric pressures", Combustion and Flame, vol. 162, pp. 3562-3568, $2015 \mathrm{~b}$. 